\title{
Tuning Yu-Shiba-Rusinov States in a Quantum Dot
}

\author{
Anders Jellinggaard, Kasper Grove-Rasmussen, Morten Hannibal Madsen, and Jesper Nygård \\ Center for Quantum Devices, University of Copenhagen
}

(Dated: July 23, 2018)

\begin{abstract}
We present transport spectroscopy of sub-gap states in a bottom gated InAs nanowire coupled to a normal lead and a superconducting aluminium lead. The device shows clearly resolved subgap states which we can track as the coupling parameters of the system are tuned and as the gap is closed by means of a magnetic field. We systematically extract system parameters by using numerical renormalization group theory fits as a level of the quantum dot is tuned through a quantum phase transition electrostatically and magnetically. We also give an intuitive description of sub-gap excitations.
\end{abstract}

\section{INTRODUCTION}

Hybrid superconductor-quantum dot devices ${ }^{1}$ are heavily employed in recent experimental programs. For instance, quantum dots serve as an integral component of proposals to form ${ }^{2,3}$, manipulate ${ }^{4-6}$, and probe ${ }^{7-9}$ Majorana bound states ${ }^{10,11}$. In Cooper pair splitters, the dynamics of quantum dots filter local Andreev reflections from the desired non-local Andreev reflections to form a source of entangled electrons. ${ }^{12-14}$

In a dot-superconductor system, where the charging energy is larger than the order parameter, quasiparticles in the superconductor bind to the dot by the exchange interaction and give rise to sub-gap excitations. ${ }^{15}$ When these quasiparticles form a singlet with electrons on the dot, the resultant states are called Yu-Shiba-Rusinov states $^{16-18}$ and have historically been investigated primarily through scanning tunneling microscopy ${ }^{19,20}$. Only recently, have these excitations been observed in transport experiments. ${ }^{21-33}$ We will give an intuitive description of sub-gap excitations in the following section.

To experimentally investigate sub-gap excitations, we fabricated a bottom gated normal metal/nanowire/ superconductor device (N-NW-S), which allows for the formation of a gate defined quantum dot proximitized to the superconductor. The device shows clearly resolved sub-gap states that we can track as the device is electrostatically tuned. In this way we follow a single charge state of the dot, through the doublet to singlet quantum phase transitions occurring as the barrier to the superconductor is lowered. We fit measured excitations energies to a simulation developed for this purpose using the non-perturbative numerical renormalization group (NRG) method $^{32,34,35}$, and in this way systematically extract physical parameters of the device.

The system investigated is in many ways similar to NNW-S devices where Majorana bound states have been examined, ${ }^{36-38}$ and a good understanding of the magnetic field behavior of proximitized nanowire quantum dots is necessary to understand transport data of these similar devices. We probe in detail the magnetic field behavior and observe excitations apparently clinging to zero bias as the gap is about to close, consistent with a recent experiment ${ }^{21}$.

\section{A. Sub-gap states}

We consider a quantum dot described by the Anderson model (full hamiltonian in Appendix. B) with a single level at $\epsilon$ and a charging energy of $U$, coupled to a normal lead and to a superconducing lead with order parameter $\Delta$. The strength of the coupling to lead $\alpha$ ( $\alpha=N, S$ ), is governed by the corresponding tunneling density of states, $\Gamma_{\alpha}=2 \pi\left|t_{\alpha}\right|^{2} \nu_{F}$, where $t_{\alpha}$ is the tunneling coefficient of lead $\alpha$ and $\nu_{F}$ is the density of states of lead $\alpha$ near the Fermi level. Our data is collected in a regime where $\Gamma_{N}$ is small compared to the other energies of the system, so we consider the normal lead to be a tunnel probe ${ }^{39}$ which is used to probe the quantum dot/superconductor system.

The nature of sub-gap excitations in such a system depends on the relative size of $\Delta$ and $U \cdot{ }^{15}$ If $\Delta$ is large, the system can be understood in terms of repeated Andreev reflections giving rise to Andreev bound states. ${ }^{40}$ When $U$ is large, Andreev reflections are supressed, and instead we need to think in terms of quasiparticles (bogoliubons) in the superconducting lead. We will here develop an intuition for excitations in this case.

First, for vanishing $\Gamma_{S}$, we know exactly what the eigenstates of the model are, and we will be focusing, in particular, on the states shown in Fig. 1. These are the lowest energy singlets and doublets (only half the doublets are shown) for different values of $\epsilon$. The illustrated states are

$$
\begin{aligned}
|A\rangle & =|0\rangle & |D\rangle & =\gamma_{\uparrow}^{\dagger}|0\rangle \\
|B\rangle & =\frac{1}{\sqrt{2}}\left(\gamma_{\downarrow}^{\dagger} d_{\uparrow}^{\dagger}-\gamma_{\uparrow}^{\dagger} d_{\downarrow}^{\dagger}\right)|0\rangle & |E\rangle & =d_{\uparrow}^{\dagger}|0\rangle \\
|C\rangle & =d_{\uparrow}^{\dagger} d_{\downarrow}^{\dagger}|0\rangle & |F\rangle & =d_{\uparrow}^{\dagger} d_{\downarrow}^{\dagger} \gamma_{\uparrow}^{\dagger}|0\rangle,
\end{aligned}
$$

where we have defined $\gamma_{\sigma}^{\dagger}$ as the operator that creates the lowest energy bogoliubon ${ }^{41}$ with spin $\sigma$, and $d_{\sigma}^{\dagger}$ as the operator which creates an electron on the dot with spin $\sigma$. The figure also shows the energy of relevant excitations between these states.

As $\Gamma_{S}$ is turned up, the singlet states are mixed resulting in avoided crossings, and the same happens for the different doublet states. For instance, the coupling between $|A\rangle$ and $|B\rangle$ causes the excitation energy inside the gap to move down towards the center of the gap. The 


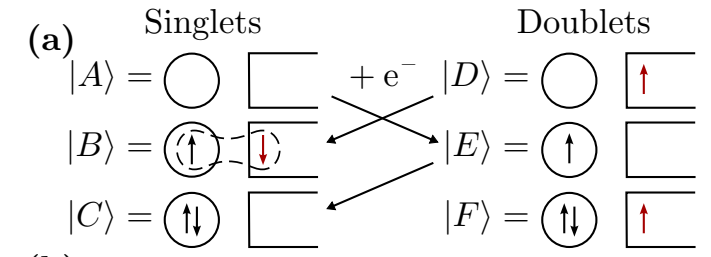

(b)
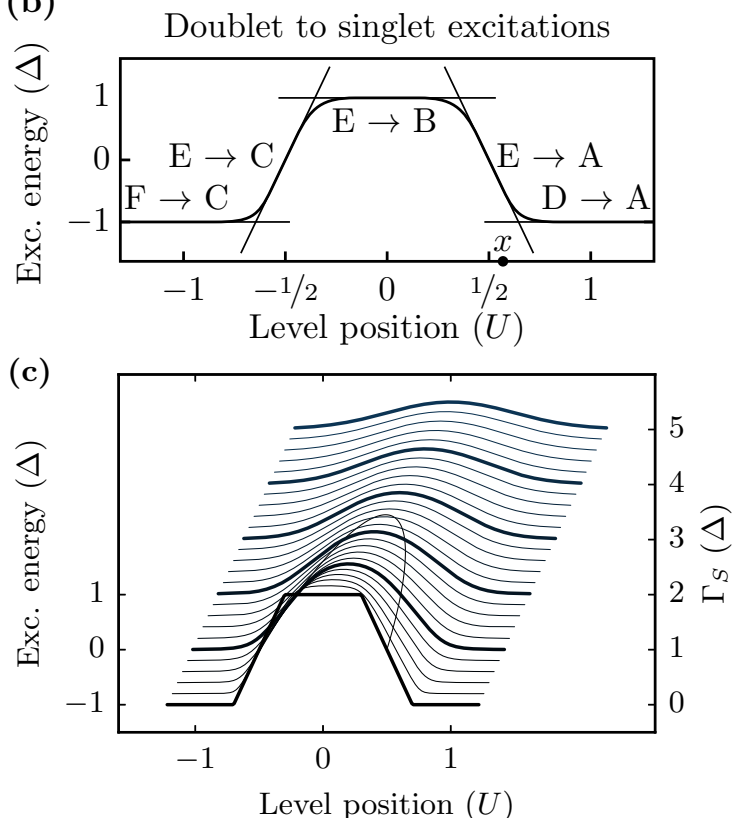

Figure 1. Excitations between states in a quantum dot/superconductor system. (a) The states under consideration with arrows in circles representing electrons in the dot and arrows in open rectangles representing bogoliubons. The dashed shape in the diagram for $|B\rangle$ depicts a singlet correlation. The arrows annotated with $+\mathrm{e}^{-}$show dominant sequential tunneling processes for transport going from $\mathrm{N}$ to $\mathrm{S}$ at the level position marked $x$ in (b). (b) A schematic diagram showing sub-gap excitations in the system, with and without anti-crossings induced by the coupling between the dot and superconductor. (c) NRG simulations of the lowest doublet to singlet transitions for different values of the coupling density of states, $\Gamma_{S}$. For all curves in (c), we have $U=5 \Delta$. The curve going across the traces mark an excitation energy of zero. Traces have been offset for clarity as indicated on the right-hand axis.

other bogoliubons (those of higher energy) will all move the sub-gap excitation in the same direction.

Eventually, this simple picture breaks down, as states with more than one bogoliubon become a significant factor in forming the low energy eigenstates. For higher $\Gamma_{S}$, it is not possible to find a simple theory that covers the entire range of $\epsilon$ and lends itself to a clear physical understanding, and one has to resort to numerical procedures. In this vein, Fig. 1(c) shows the lowest energy doublet to singlet excitation as a function of the level position and $\Gamma_{S}$ as found using NRG simulations. In the middle of the Coulomb valley the doublet to singlet excitation energy decreases with increasing $\Gamma_{S}$, indicating a stabilization of the singlet state, and eventually the energy crosses zero, which is an example of a second order quantum phase transition. ${ }^{21,42}$ For larger $\Gamma_{S}$, the ground state remains a singlet for all level positions, even as the expected number of electrons on the dot changes by 2 .

The NRG method has been applied to the proximitized Kondo model, ${ }^{43,44}$ the proximitized Anderson model, ${ }^{45}$ and to the normal metal/quantum dot/superconductor system $^{46,47}$ in the literature, and generally recreates the features seen in real systems fairly accurately, as our fits below also indicate. Note, that we are using a newly developed NRG program which does not exploit symmetries in the system to speed up the algorithm. ${ }^{48}$ Consequently, we only keep 160 states from each link of the chain. In Appendix C we compare results from our program to the phase diagram in Ref. 47 to show that there is reasonable agreement between the output from our program and that of an established program running a simulation with more states retained.

\section{B. Transport}

We imagine that transport in the device is primarily sequential in electrons tunneling from the $\mathrm{N}$ electrode to the dot-S system. This is possible once states with different numbers of fermions are mixed. In Fig. 1(a) we have tried to illustrate the dominant sequential transport processes moving electrons from $\mathrm{N}$ to $\mathrm{S}$ when the level position is near $1 / 2 U$, i.e. at $x$ in Fig. 1(b). In this case, considering again - artificially - only one bogoliubon state, the lowest energy singlet, $|s\rangle$, is a linear combination of primarily $|A\rangle$ but with some weight on $|B\rangle$ and $|C\rangle$, and the lowest energy doublet, $|d\rangle$ consist mainly of $|E\rangle$ with some weight on $|D\rangle$ and $|F\rangle$. Transport occurs by repeatedly swapping the state between $|s\rangle$ and $|d\rangle$ by adding electrons to the dot from the $\mathrm{N}$ lead.

Fermi's golden rule tells us that the rate at which we go from $|s\rangle$ to $|d\rangle$ is proportional to $\left|\left\langle d\left|d_{\uparrow}^{\dagger}\right| s\right\rangle\right|^{2}$ which, for low $\Gamma_{S}$, is close to what we would expect for a nonproximitized dot. Going from $|d\rangle$ to $|s\rangle$ occurs at a rate proportional to $\left|\left\langle s\left|d_{\uparrow}^{\dagger}\right| d\right\rangle\right|^{2}$, which is smaller because only terms involving $|B\rangle$ and $|D\rangle$ or $|C\rangle$ contribute, c.f. Fig. 1(a). Intuitively, we have to move two electrons across the barrier to $\mathrm{S}$ in this transport process.

\section{EXPERIMENTAL RESULTS}

The device is a bottom gated $70 \mathrm{~nm}$ diameter InAs nanowire with one $\mathrm{Ti} / \mathrm{Au}$ contact and one $\mathrm{Ti} / \mathrm{Al}$ contact approximately $330 \mathrm{~nm}$ apart. The bottom gates have a $55 \mathrm{~nm}$ center-to-center distance and are separated from the nanowire by a $24 \mathrm{~nm} \mathrm{HfO}_{2}$ dielectric. The contacts are both well coupled to the nanowire compared to the deliberate transport barriers we impose with the bottom gates to form the dot, and the $\mathrm{Ti} / \mathrm{Al}$ contact is supercon- 


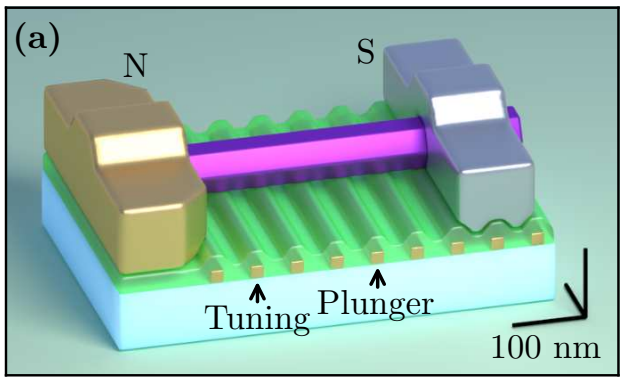

(b)

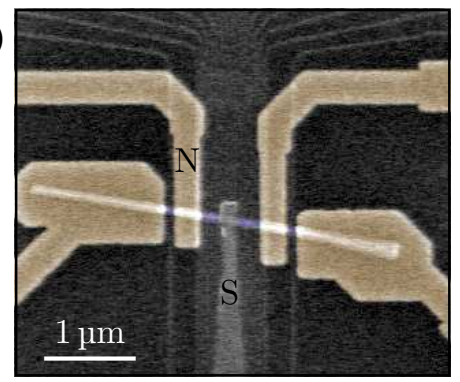

Figure 2. (a) Artist impression of a $0.6 \mu \mathrm{m} \times 0.4 \mu \mathrm{m}$ cutout of the device, to scale. The model shows the surface of the $\mathrm{SiO}_{2}$ substrate, bottom gates, insulating $\mathrm{HfO}_{2}$ (shown in green), InAs nanowire, gold contact, and aluminium contact. Details are in Appendix A. We assign names to two of the gates as shown. (b) SEM micrograph of a lithographically similar device. Note, that only the part of the device between the gold electrode, $\mathrm{N}$, and the aluminium electrode, $\mathrm{S}$, is used.

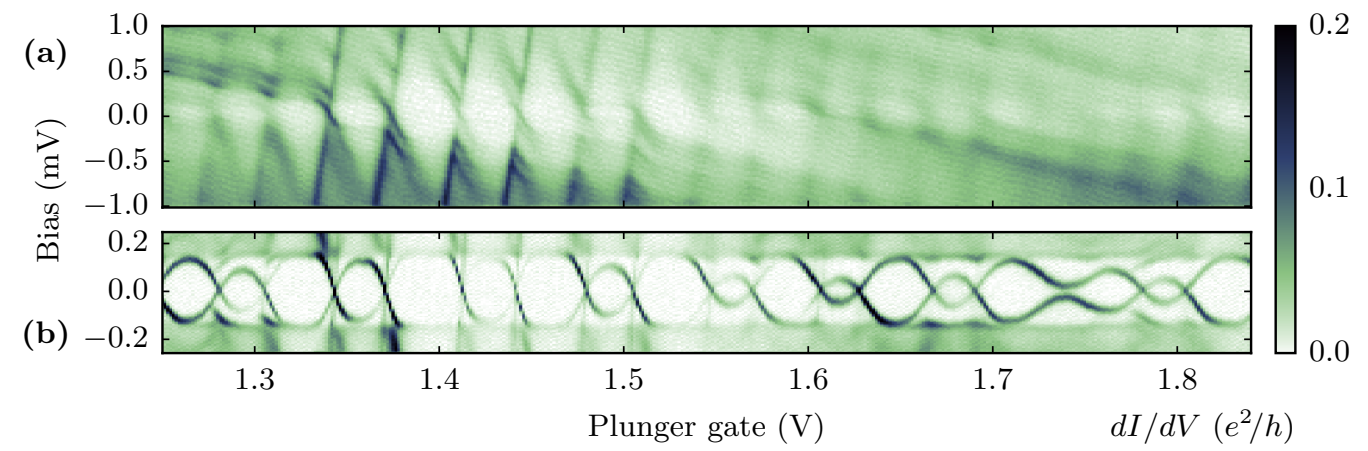

Figure 3. Differential conductance at $35 \mathrm{mK}$ with and without an externally applied $150 \mathrm{mT}$ in-plane field. The field drives the aluminium contact normal in (b). Regions that show heavily tunnel-broadened Coulomb diamonds also show sub-gap excitations far inside the gap when the $\mathrm{Al}$ contact is superconducting.

ducting with $\Delta=0.14 \mathrm{mV}$. Further fabrication details can be found in Appendix A.

Figure 2(a) shows a scale model of our device and our designation of a "tuning gate", $V_{T}$, and a "plunger gate", $V_{P}$. A scanning electron microscopy (SEM) micrograph of a similar device is shown in Fig. 2(b), where only the nanowire segment between the $\mathrm{N}$ and $\mathrm{S}$ electrodes is probed by transport. In all plots, we apply a bias, $V_{s d}$ to the aluminium contact and measure differential conductance, $d I / d V$, through the device at a temperature of $35 \mathrm{mK}$. Figure 3(a) shows typical transport data with the aluminium contact driven normal by a field, and Fig. 3(b) shows corresponding bias spectroscopy at zero field where the superconducting gap is visible as a horizontal band of low differential conductance between $V_{s d}=-0.14 \mathrm{mV}$ and $0.14 \mathrm{mV}$. The normal state data shows the usual Coulomb diamonds for $V_{P}<1.6 \mathrm{~V}$, but for $V_{P}>1.6 \mathrm{~V}$ these diamonds become difficult to resolve, as the excitations are heavily tunnel broadened by the coupling the aluminium contact. In regions where the excitations are broadened in the normal state data (cf. Fig. 3(a)), which we attribute to a strong coupling to the aluminium contact, we see that the sub-gap excitations in Fig. 3(b) are pushed far inside the gap. In the remainder of this article, we investigate how these sub-gap excitations respond to gate tuning and to small (less than $B_{c}$ ) magnetic fields.

\section{A. Gate tuning}

Figure 4 shows the zero bias differential conductance of the device as a function of the potential, $V_{P}$ and $V_{T}$, of the plunger and tuning gate. Both gates couple to the dot and have capacitances of $C_{P} \approx 5.5 \mathrm{aF}$ and $C_{T} \approx$ $3 \mathrm{aF}$ (lever arms $\alpha_{P} \approx 0.035$ and $\alpha_{T} \approx 0.02$ ) for the plunger and tuning gates, respectively. We define $V_{P}^{\prime}=$ $V_{P}+\left(V_{T}-0.3 \mathrm{~V}\right) \times 0.57$ to compensate for this cross capacitance, and will use this for all subsequent figures instead of $V_{P}$. Note the overall increase in conductance for increasing $V_{T}$, which we ascribe to a lowering of the barrier to the normal lead consistent with the position of the tuning gate. Later, we shall see that $\Gamma_{N}$ depends on $V_{T}$ exponentially, which supports this assertion. Also evident in these plots is a quantum phase transition (at the $*$ ), which will become clearer in later plots.

Figure 5 shows how the sub-gap excitations respond to tuning, and a few trends are apparent going from low $\left(a_{0}\right)$ to high $\left(a_{6}\right) V_{T}$. First, we see again the overall increase in conductance with higher $V_{T}$. Secondly, as $V_{T}$ is changed, the sub-gap excitations of Fig. 5 shift in energy, with no overall trend, which we interpret as 


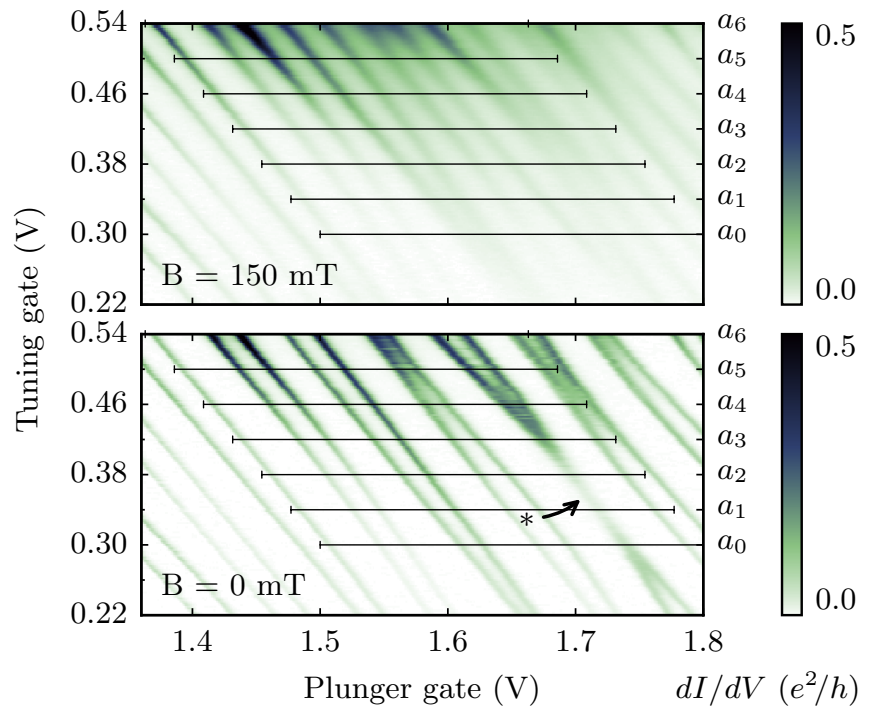

Figure 4. Conductance at zero bias as a function of the tuning gate and the raw plunger gate potential, with and without a magnetic field driving the aluminium contact normal. The lines in these plots shows the cuts done by $a_{0}-a_{6}$ of Fig. 5 . For certain configurations of the tuning gate, the ground state remains a singlet as a dot level is brought past the fermi-level with the plunger gate, and this is evident at the $*$.

mesoscopic fluctuations of $\Gamma_{S}$ as the wavefunctions of the dot states are perturbed by the changing $V_{T}$ and $V_{P}$. The quantum phase transition is very clear in this plot, occurring for the charge state labeled IV around $V_{T}=$ $0.44 \mathrm{~V}$, i.e. between $a_{3}$ and $a_{4}$. The other charge states do not undergo this kind of quantum phase transition in the data shown. We point out, that the transitions are not significantly tunnel-broadened compared to the size of the gap, so we can assume that normal lead is weakly coupled to the dot.

To extract quantitative parameters for the system, we fit a model to the data consisting of single levels independently interacting with the superconductor, such that each level is described by the proximitized Anderson model. In this model, each level is described by the following parameters: The charging energy $U$, the potential of the plunger gate at the center of the corresponding Coulomb valley $V_{0}$, the plunger gate capacitance $C_{P}$, and the coupling strengths $\Gamma_{S}$ and $\Gamma_{N}$. We will find quantitative estimates for all these parameters.

Specifically, we find $U$ from the height of the corresponding Coulomb diamond in Fig. 3(a), and we find $V_{0}$ by looking at Fig. 5 . We initially assume $\Gamma_{N}$ is weak, in which case it has little effect on level positions and does not drive the NW-S system out of equilibrium, and we find $C_{P}$ and $\Gamma_{S}$ using one of two methods both involving a fit based on the NRG method: for Method-1, we find $C_{P}$ from the normal state data in Fig. 4 and use $\Gamma_{S}$ as a fitting parameter to fit the observed level positions. For Method-2, we use both $C_{P}$ and $\Gamma_{S}$ as fitting parameters. Fits to two of the datasets are shown in Fig. 5 for both
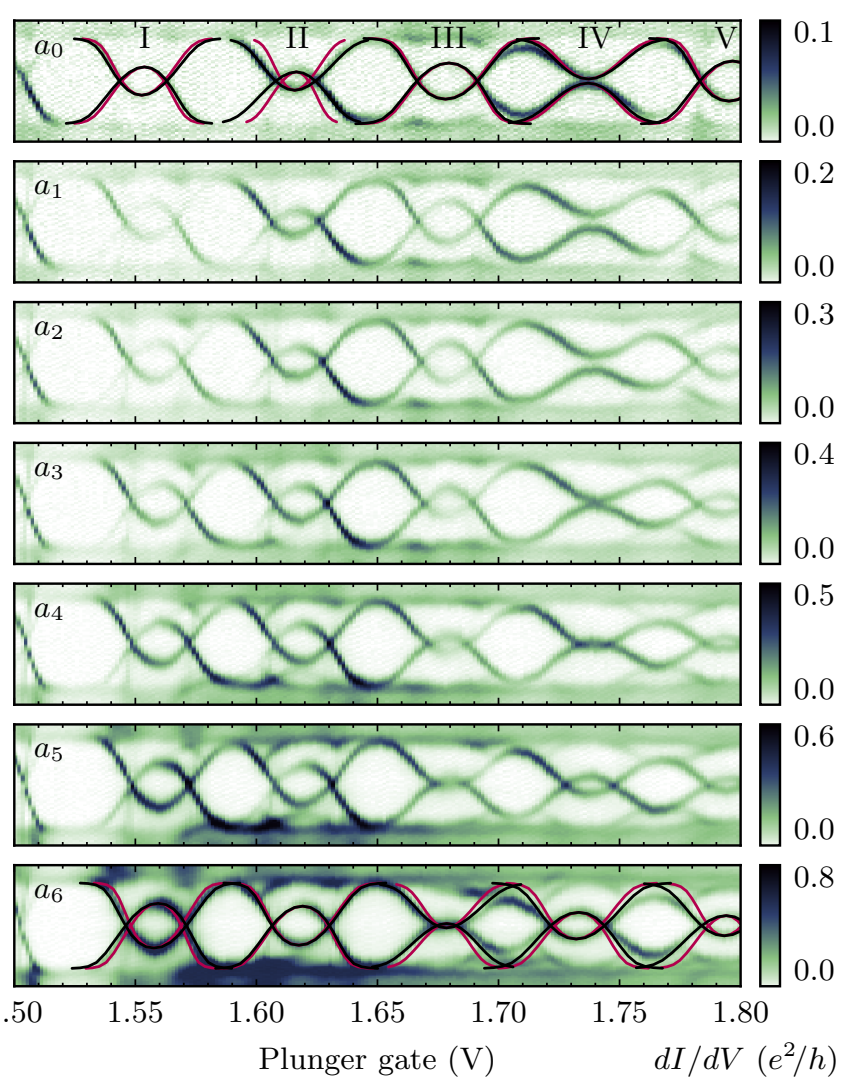

Figure 5. Bias spectroscopy of the sub-gap states for different gate configurations. In every plot, the y-axis is the potential of the superconducting lead relative to the normal lead and ranges from $-0.2 \mathrm{mV}$ to $0.2 \mathrm{mV}$. Note the different $d I / d V$ scales. In the plot $a_{n}$, the tuning gate is set to $(300+40 \mathrm{n}) \mathrm{mV}$. We adjust for cross capacitance as described in the text. Example NRG fits are overlaid plots $a_{0}$ and $a_{6}$. The red curves are made using Method 1 and the black curves are made using Method 2, see text for details.

methods, the rest are included in the supplementary information.

Generally both methods reproduce the gate dependence of the sub-gap state excitations well. The most significant divergence is around $V_{P}^{\prime}=1.70 \mathrm{~V}$ and $V_{P}^{\prime}=$ $1.76 \mathrm{~V}$, where additional excitation lines are present inside the gap. The presence of these lines suggest that the levels are not independent in this region.

Having found the values of $\Gamma_{S}$ at each level crossing from our NRG fits, we extract $\Gamma_{N}$ from the conductance at each Coulomb peak when the superconductor is driven normal by an external magnetic field, i.e. from the data in Fig. 4. Specifically

$$
G_{\text {peak }}=\frac{e^{2}}{h} \frac{4 \Gamma_{S} \Gamma_{N}}{\left(\Gamma_{S}+\Gamma_{N}\right)^{2}},
$$

where $G_{\text {peak }}$ is the maximal conductance of the device at the Coulomb peak. ${ }^{49}$ The values of $\Gamma_{N}$ and $\Gamma_{S}$ that we extract are shown in Fig. 6. $\Gamma_{N}$ shows an exponential dependence on the tuning gate potential, as expected 


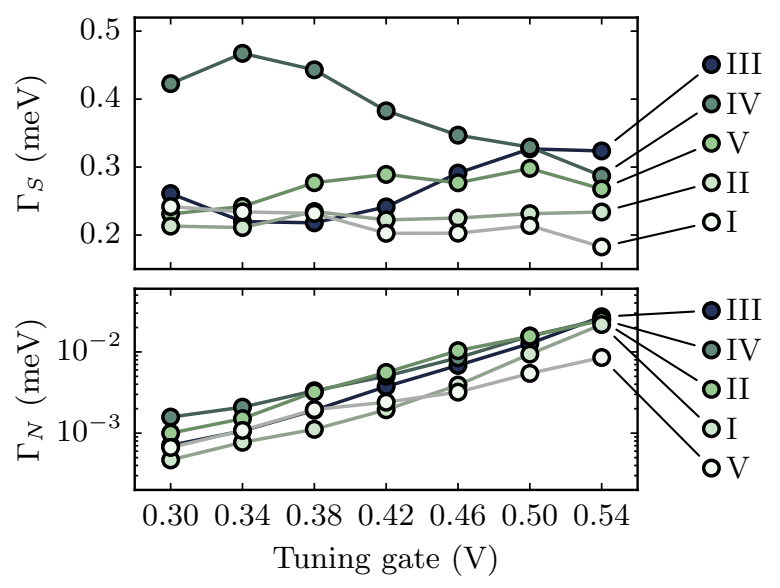

Figure 6. Coupling strengths $\Gamma_{S}$ and $\Gamma_{N}$ of each level transition shown in Fig. 5. We extract $\Gamma_{S}$ from our NRG fits, and use the conductance on resonance in the $B=150 \mathrm{mT}$ data of Fig. 5 to find $\Gamma_{N}$. There is one trace in the plot for each level transition, and the roman numerals refer back to the labels in Fig. 5.

for an electron tunneling through a potential barrier. In contrast, $\Gamma_{S}$ varied non-monotonically and did not have a systematic dependence on gate. Therefore, we attribute the variations we do see in $\Gamma_{S}$ to mesoscopic fluctuations caused by perturbations of the dot wavefunctions, rather than a changing potential barrier.

\section{B. Results: Behavior at Field}

We now turn to the magnetic field dependence of the sub-gap states. Figure 7 shows what happens to the plot $a_{1}$ in Fig. 5 as a field is applied in the plane of the sample in a direction perpendicular to the nanowire. As the field increases, the doublet states Zeeman split, which is clear where the ground state is a singlet. When the ground state is a doublet, only one excitation is possible from the ground state, and only one peak is seen in transport. ${ }^{21}$ When analysing our data, we will augment the Anderson model hamiltonian of the dot from the introduction with a Zeeman term of the form

$$
H_{Z}=g \mu_{B} B \cdot S,
$$

where $g$ is the g-factor of the level, $\mu_{B}$ is the Bohr magneton, $B$ is the strength of the magnetic field, and $S$ is the spin of the dot; note that we always align the $z$-axis of the spin basis with the magnetic field. In our quantum dot, the effective g-factor varies significantly between levels, ${ }^{50}$ and even within a single level. For instance, in the charge state labeled II in Fig. 7, the splitting of the excitations line left of center (near more negative $V_{P}$ ) is very different from the splitting right of center.

At the charge state labeled IV in Fig. 5 we are able to induce a quantum phase transition by a applying a magnetic field, so we focus on this level crossing. Figure 8
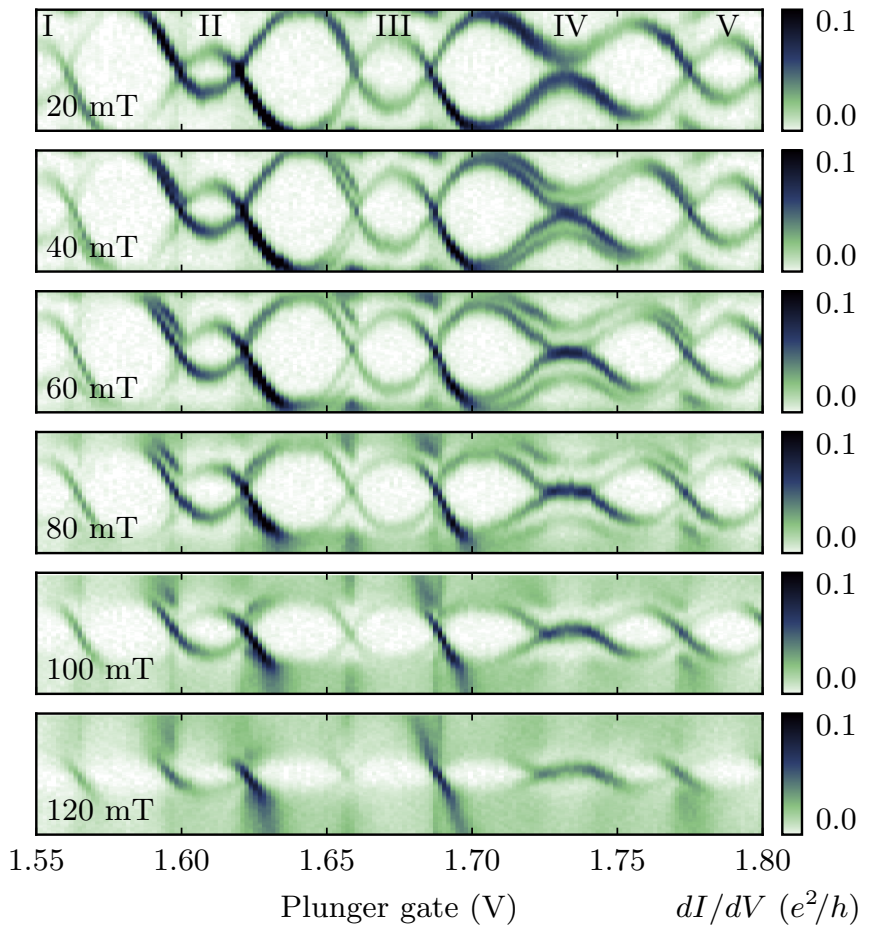

Figure 7. Evolution of the sub-gap states as the gap closes upon the application of an external field in plane with the substrate perpendicular to the nanowire. The tuning gate is set to $V_{T}=0.34 \mathrm{~V}$ in all these plots, and the bias range is $\pm 125 \mu \mathrm{V}$. The roman numerals on odd charge states refer back to the labels in Fig. 5 .

shows the dependence of transport at the center of the crossing both as the field magnitude is increased and as the field is rotated. As in our other dataset, we again note the absence of a transition from the excited member of the doublet to the singlet, which is what causes the peaks in Fig. 8 for $V_{T}>0.42 \mathrm{~V}$ to only move in one direction instead of splitting.

As is apparent in the plots $c_{0}$ through $c_{5}$ of Fig. 8, the $\mathrm{g}$-factor of our system shows a high degree of anisotropy. This is a common property of quantum dots in InAs nanowires ${ }^{50,51}$ and was also addressed by Lee et al. for YSR states. ${ }^{21}$

In the bias vs. field strength plots of Fig. 8, specifically plots $b_{0}, b_{1}$, and $b_{2}$, we note that the excitation of the doublet that moves down in energy has an apparent tendency to stick to zero bias. This effect has been observed before and can be understood in terms of a level repulsion from the gap states as the gap closes, pinning the excitations near zero energy. ${ }^{21}$

We estimate the level positions from the data plotted in $c_{1}$ of Fig. 8, and fit the g-factor at angles of $0 \mathrm{rad}$, $0.9 \pi \mathrm{rad}$, and $1.4 \pi \mathrm{rad}$ using our NRG model. The latter two angles correspond to minimal and maximal Zeeman splitting, note that the splitting at $0.9 \pi \mathrm{rad}$ is hard to estimate precisely. For these angles, we find g-factors of approximately 22,8 , and 23 respectively. 
Adjusted plunger gate at $1.74 \mathrm{~V}$
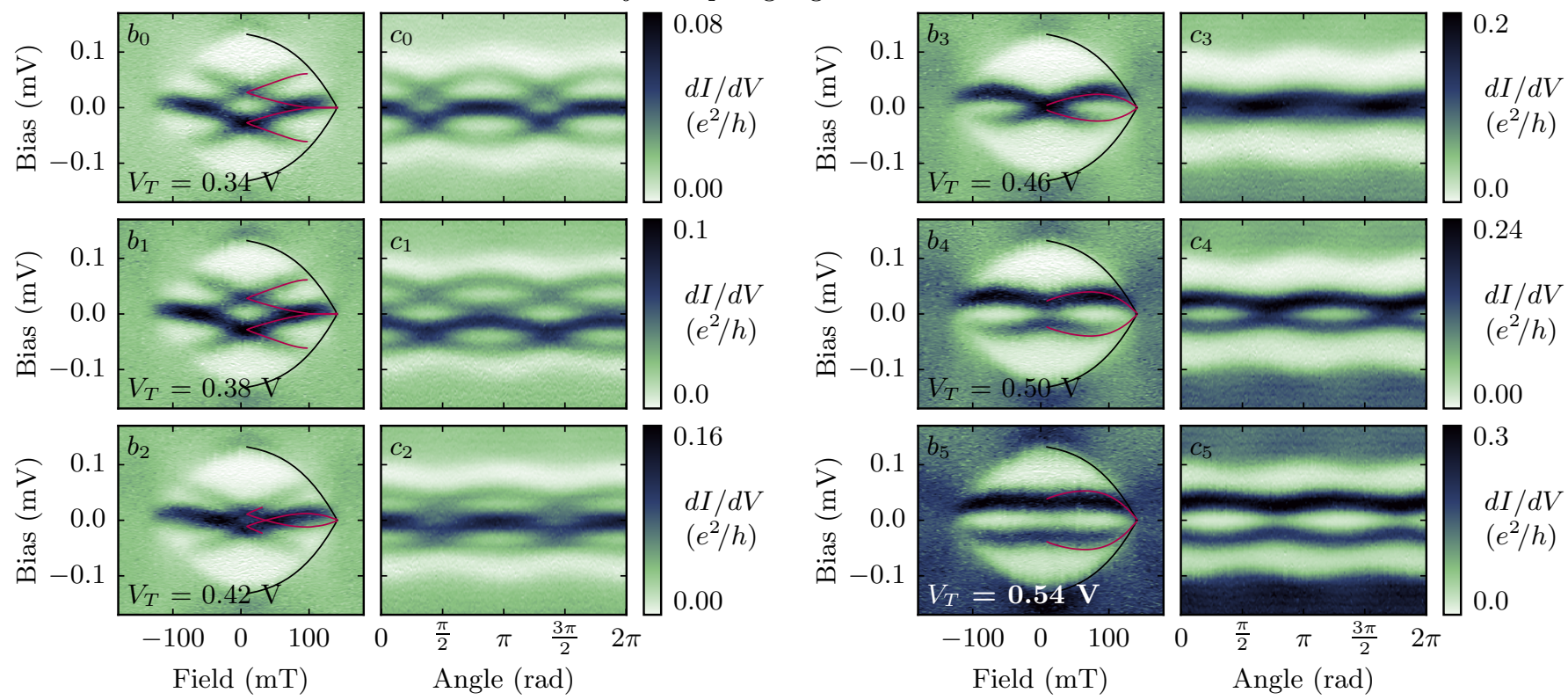

Figure 8. Magnetic field dependence of sub-gap transport in the center of a level transition for different values of the tuning gate potential. In the data sets $b_{0}$ through $b_{5}$ the field was applied in the plane of the sample perpendicular to the nanowire, and in the data sets $c_{0}$ through $c_{5}$ the field was held at $60 \mathrm{mT}$ and rotated in the plane of the sample with a direction of 0 radians being perpendicular to the wire. The data has been corrected for a drifting zero-bias across the device as detailed in the supplementary information. The plots also show data from an NRG simulation, specifically the allowed excitations from the ground states of the system (red lines). The black lines show a phenomenological model of the gap closing used as an input to the simulation. Additional input to the simulation includes the $\Gamma_{S}$ values from Fig. 6 and a g-factor found by fitting the plot $c_{1}$ at 0 radians.

We use the g-factor at $0 \mathrm{rad}$ along with the values of $\Gamma_{S}$ found for each tuning gate value earlier, to simulate how the states split with applied field, i.e. to recreate the level positions seen in the plots $b_{0}$ through $b_{5}$. The resulting level transitions are plotted in the figure and show good agreement with the data. We plot excitations from the ground state only, but in the plot $b_{2}$, transport is also possible from the doublet state, presumably because the doublet is thermally excited.

\section{CONCLUSION}

The device presented in this paper had two features that complement each other: Transparent contacts and well coupled bottom gates with a large admissible voltage range. This made it feasible to make completely gate defined contact barriers in the device, and tune coupling parameters over a large range while keeping mostly single-dot behavior. In combination with well resolved sub-gap states, the device provided an excellent platform to study the dependence of Yu-Shiba-Rusinov states to $\Gamma_{S}$-tuning and to magnetic fields. Future studies may involve testing the recent theoretical predictions that the singlet-doublet phase diagram is modified by the normal metal coupling. ${ }^{47}$

For the data presented in this paper, we used a gate between the quantum dot and the normal contact to tune our device. This had a large effect on $\Gamma_{N}$ which in turn has only a small effect on level positions. On the other hand, mesoscopic fluctuations of $\Gamma_{S}$ (on the other side of the device) caused by this tuning, has a large and, apriori, unpredictable effect on $\Gamma_{S}$. Effects like this can appear in gated quantum dot devices, whether it involves a superconducting contact or not, but this device is an interesting example as the two contact barriers influence transport in very dissimilar ways.

Modeling the device using the proximitized Anderson model by means of the NRG method yielded excitation energies in good agreement with our data, and the coupling parameters extracted from these fits follow the potentials of the bottom gates in a physically reasonable way. The behavior under magnetic field is entirely consistent with a simple Zeeman splitting in combination with the gap closing. This behavior has been described before. ${ }^{21}$ However, here we model this scenario quantitatively using the NRG method starting from parameters determined at zero field, and show good agreement with observed data. 


\section{ACKNOWLEDGMENTS}

We would like to thank Ramon Aguado and Jens Paaske for helpful discussions and Peter Krogstrup, Claus B. Sørensen and Erik Johnson for experimental contributions. Funding for this project was provided by the EU FP7 project SE2ND (Source of Entangled Electrons in Nano Devices), the Carlsberg Foundation, the Danish Research Council DFF-FNU and the Danish National Research Foundation.

\section{Appendix A: Fabrication details}

The bottom gates were fabricated on a Si substrate with $500 \mathrm{~nm}$ oxide, and are composed of $5 \mathrm{~nm} \mathrm{Ti}$ and $12 \mathrm{~nm} \mathrm{Au}$. These gates have a center to center distance of $55 \mathrm{~nm}$. The gates are covered with $24 \mathrm{~nm} \mathrm{HfO}_{2}$ deposited by atomic layer depositioning. This $\mathrm{HfO}_{2}$ is deposited in three $8 \mathrm{~nm}$ layers of successively smaller extent to avoid fencing, where the oxide does not break off cleanly where it meets resist walls and instead stand proud off the surface after lift-off.

$70 \mathrm{~nm}$ diameter InAs nanowires were deposited from a suspension in isopropanol. In the evaporation chamber, immediately prior to metalization of each contact, argon ion milling was used to remove the native oxide from the nanowire. The Au contact uses a $10 \mathrm{~nm}$ Ti sticking layer, and the $\mathrm{Al}$ contact uses a $5 \mathrm{~nm} \mathrm{Ti}$ sticking layer. Compared to the data shown in this article, the device is significantly more conductive when a higher potential is applied to the bottom gates, suggesting that the tunnel barriers seen in the data are gate defined as opposed to contact defined.

Tuning the potentials of the gates allow the device to be operated in different regimes; in this paper we focus on single dot behavior by forming a central potential dip (see Fig. 9). We note that bottom gates under a contact, for instance the second bottom gate from the right in Fig. 2, generally do not show any significant effect on transport through the device. This suggest that the gates are strongly screened, or that the contacts - by diverting current out of the wire already very near the edge of the contact-make it a moot point whether the sections of nanowire above these gates are depleted or not.

The device investigated in this paper forms part of a larger two-sided device. To avoid complications from the other side of the device, this part of the nanowire was electrostatically depleted during measurements.

\section{Appendix B: Model details}

For the discussions in the article and in our models, we use the following hamiltonian

$$
H=H_{d}+H_{S}+H_{t \mathrm{~S}}+H_{t \mathrm{~N}},
$$

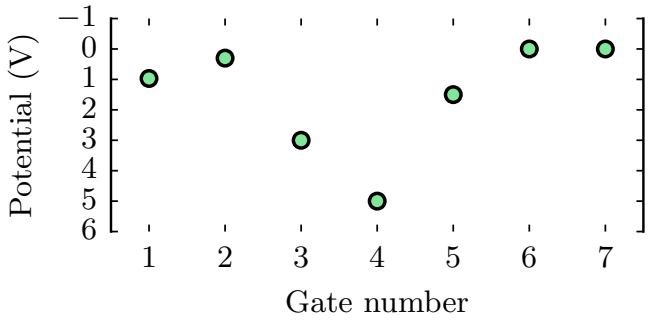

Figure 9. Electric potentials applied to each relevant bottom gate. The gates are numbered starting from the gold side of the device (left side in Fig. 2). Gate nr. 2 we call the tuning gate and gate nr. 5, which is more strongly coupled to the energy levels of the dot, we call the plunger gate.

with each part given below. The quantum dot has the hamiltonian

$$
H_{d}=\sum_{\sigma} \epsilon_{\sigma} c_{\sigma}^{\dagger} c_{\sigma}+\frac{U}{2}\left(\sum_{\sigma} c_{\sigma}^{\dagger} c_{\sigma}-1\right)^{2}
$$

where $c_{\sigma}^{\dagger}$ creates an electron with spin $\sigma$ on the dot, $U$ is the charging energy of the dot, and $\epsilon$ is the the single particle energy of the dot. The dot is coupled to the two leads by

$$
\begin{aligned}
& H_{t \mathrm{~S}}=\sum_{k \sigma} t_{\mathrm{S}} c_{\sigma}^{\dagger} c_{k \sigma}+H C \\
& H_{t \mathrm{~N}}=\sum_{k \sigma} t_{\mathrm{N}} c_{\sigma}^{\dagger} f_{k \sigma}+H C,
\end{aligned}
$$

where $c_{k \sigma}^{\dagger}$ creates an ordinary fermion in the superconducting lead with momentum $k$ and spin $\sigma, f_{k \sigma}^{\dagger}$ creates a quasiparticle in the normal lead, and the $t$ 's are tunneling coefficients assumed spin and momentum independent. The hamiltonian of the superconductor is

$$
H_{S}=\sum_{k \sigma} \xi_{k \sigma} c_{k \sigma} c_{k \sigma}^{\dagger}+\sum_{k}\left(\Delta c_{k \uparrow} c_{-k \downarrow}+\Delta c_{-k \downarrow}^{\dagger} c_{k \uparrow}^{\dagger}\right)
$$

where $\Delta$ is the order parameter of superconductor which we assume is real.

For our NRG simulations, we assume that $t_{\mathrm{N}}$ and the temperature of the system are negligible, we discretize the leads logarithmically using a discretization factor, $\Delta$, of 2.5, and map the system to a chain of fermions starting with the quantum dot. We add sites of the chain one at a time, and at each step retain the lower 160 eigenstates. The sub-gap excitation energies converge quickly, ${ }^{43}$ so we only extend the chain to 25 sites. To simulate the gap closing with applied field, we created, by hand, a table of gap size as function of applied field based on the data in Fig. 8.

When calculating the peak conductance with the superconductor driven normal, in Eq. (1), we assume $k_{B} T \ll \Gamma_{S}+\Gamma_{N}$ consistent with our findings, and use a result from the supplementary information of Ref. 49 . 


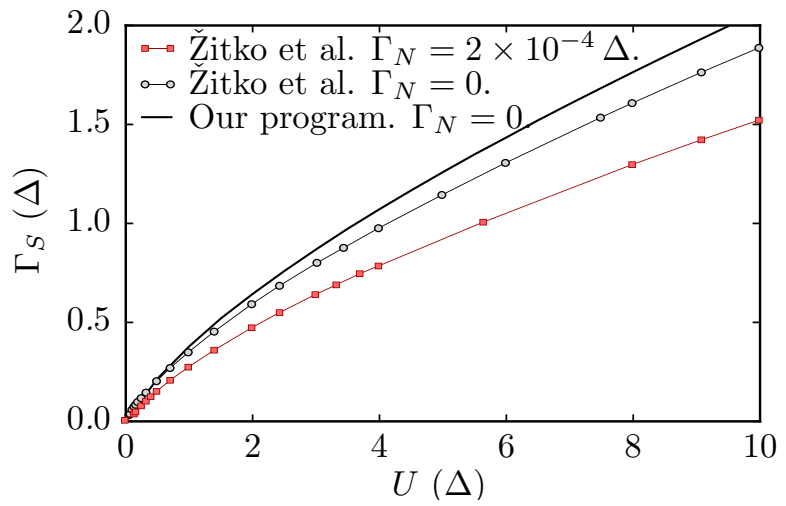

Figure 10. This figure compares output from our NRG program to Fig. 1 in Ref. 47.

\section{Appendix C: Evaluating the NRG program}

Since we are not exploiting symmetries in our NRG program, we cannot keep as many states as others do in the calculations. Therefore we compared the output of our program to a plot in Žitko et al, Physical Review B 91, 045441 (2015) (our Ref. 47) showing the quantum phase transition in a proximitized dot (see Fig. 10). Žitko et al. also include simulations for a small coupling to the normal lead, which we reproduce in Fig. 10 to show that the error made by using our program is small compared to the error made by not considering finite $\Gamma_{N}$. Note, that our definition of $\Gamma_{S}$ differs from the one used in Žitko et al. by a factor of two, so we scaled ours for this plot.
1 S. De Franceschi, L. Kouwenhoven, C. Schönenberger, and W. Wernsdorfer, Nature nanotechnology 5, 703 (2010).

2 J. D. Sau and S. D. Sarma, Nature Communications 3, 964 (2012).

3 M. Leijnse and K. Flensberg, Physical Review B 86, 134528 (2012)

${ }^{4}$ K. Flensberg, Physical Review Letters 106, 090503 (2011).

5 M. Leijnse and K. Flensberg, Physical Review Letters 107, 210502 (2011)

6 J. Alicea, Y. Oreg, G. Refael, F. von Oppen, and M. P. a. Fisher, Nature Physics 7, 412 (2011).

7 M. Leijnse and K. Flensberg, Physical Review B 84, 140501 (2011)

8 Y. Cao, P. Wang, G. Xiong, M. Gong, and X.-Q. Li, Physical Review B 86, 115311 (2012).

9 D. E. Liu and H. U. Baranger, Physical Review B 84, 201308 (2011).

10 Y. Oreg, G. Refael, and F. von Oppen, Physical Review Letters 105, 177002 (2010).

11 J. D. Sau, R. M. Lutchyn, S. Tewari, and S. Das Sarma, Physical Review Letters 104, 040502 (2010).

12 P. Recher, E. V. Sukhorukov, and D. Loss, Phys. Rev. B 63, 165314 (2001).

13 L. Hofstetter, S. Csonka, J. Nygård, and C. Schönenberger, Nature 461, 960 (2009).

14 L. G. Herrmann, F. Portier, P. Roche, A. L. Yeyati, T. Kontos, and C. Strunk, Phys. Rev. Lett. 104, 026801 (2010).

15 G. Kiršanskas, M. Goldstein, K. Flensberg, L. I. Glazman, and J. Paaske, ArXiv e-prints (2015), arXiv:1509.02770 [cond-mat.mes-hall].

16 L. Yu, Acta Physica Sinica 21, 75 (1965).

17 H. Shiba, Progress of Theoretical Physics 40, 435 (1968).

18 A. I. Rusinov, Soviet Journal of Experimental and Theoretical Physics 29, 1101 (1969).

19 A. Yazdani, B. A. Jones, C. P. Lutz, M. F. Crommie, and D. M. Eigler, Science 275, 1767 (1997).

${ }^{20}$ K. J. Franke, G. Schulze, and J. I. Pascual, Science 332, 940 (2011).

${ }^{21}$ E. J. H. Lee, X. Jiang, M. Houzet, R. Aguado, C. M. Lieber, and S. De Franceschi, Nature Nanotechnology 9, 79 (2014).
${ }^{22}$ K. Grove-Rasmussen, H. I. Jørgensen, B. M. Andersen, J. Paaske, T. S. Jespersen, J. Nygård, K. Flensberg, and P. E. Lindelof, Phys. Rev. B 79, 134518 (2009).

23 J.-D. Pillet, C. H. L. Quay, P. Morfin, C. Bena, A. L. Yeyati, and P. Joyez, Nature Physics 6, 965 (2010).

${ }^{24}$ R. S. Deacon, Y. Tanaka, A. Oiwa, R. Sakano, K. Yoshida, K. Shibata, K. Hirakawa, and S. Tarucha, Phys. Rev. B 81, 121308 (2010).

25 T. Dirks, T. L. Hughes, S. Lal, B. Uchoa, Y.-F. Chen, C. Chialvo, P. M. Goldbart, and N. Mason, Nature Physics 7, 386 (2011)

${ }^{26}$ R. S. Deacon, Y. Tanaka, A. Oiwa, R. Sakano, K. Yoshida, K. Shibata, K. Hirakawa, and S. Tarucha, Phys. Rev. Lett. 104, 076805 (2010).

27 W. Chang, V. E. Manucharyan, T. S. Jespersen, J. Nygård, and C. M. Marcus, Phys. Rev. Lett. 110, 217005 (2013), arXiv:1211.3954 [cond-mat.mes-hall].

28 B.-K. Kim, Y.-H. Ahn, J.-J. Kim, M.-S. Choi, M.-H. Bae, K. Kang, J. S. Lim, R. López, and N. Kim, Phys. Rev. Lett. 110, 076803 (2013).

29 A. Kumar, M. Gaim, D. Steininger, A. L. Yeyati, A. Martín-Rodero, A. K. Hüttel, and C. Strunk, Physical Review B 89, 075428 (2014).

30 J. Schindele, A. Baumgartner, R. Maurand, M. Weiss, and C. Schönenberger, Phys. Rev. B 89, 045422 (2014).

31 E. J. H. Lee, X. Jiang, R. Aguado, G. Katsaros, C. M. Lieber, and S. De Franceschi, Phys. Rev. Lett. 109, 186802 (2012).

32 J.-D. Pillet, P. Joyez, R. Žitko, and M. F. Goffman, Phys. Rev. B 88, 045101 (2013).

33 R. Delagrange, R. Weil, A. Kasumov, M. Ferrier, H. Bouchiat, and R. Deblock, ArXiv e-prints (2016), arXiv:1601.03878.

34 K. Wilson, Reviews of Modern Physics 47, 773 (1975).

35 R. Bulla, T. Costi, and T. Pruschke, Reviews of Modern Physics 80, 395 (2008).

${ }^{36}$ V. Mourik, K. Zuo, S. M. Frolov, S. R. Plissard, E. P. A. M. Bakkers, and L. P. Kouwenhoven, Science (New York, N.Y.) 336, 1003 (2012).

37 A. Das, Y. Ronen, Y. Most, Y. Oreg, M. Heiblum, and H. Shtrikman, Nature Physics 8, 887 (2012). 
38 S. M. Albrecht, A. P. Higginbotham, M. Madsen, F. Kuemmeth, T. S. Jespersen, J. Nygård, P. Krogstrup, and C. M. Marcus, Nature 531, 206 (2016).

39 Recent numerical work suggest that the normal lead may have a non-pertubative effect on the system, so this approximation may not be entirely justified. ${ }^{47}$.

40 T. Meng, S. Florens, and P. Simon, Physical Review B 79, 224521 (2009).

41 M. Tinkham, Introduction to Superconductivity, 2nd ed. (Dover Publications, 2004).

42 S. Sachdev, in Handbook of Magnetism and Advanced Magnetic Materials (John Wiley \& Sons, Ltd, 2007).

43 K. Satori, H. Shiba, O. Sakai, and Y. Shimizu, Journal of the Physical Society of Japan 61, 3239 (1992).

44 O. Sakai, Y. Shimizu, H. Shiba, and K. Satori, Journal of the Physical Society of Japan 62, 3181 (1993).
45 T. Yoshioka and Y. Ohashi, Journal of the Physical Society of Japan 69, 1812 (2000).

46 Y. Tanaka, N. Kawakami, and A. Oguri, Journal of the Physical Society of Japan 76, 074701 (2007).

47 R. Žitko, J. S. Lim, R. López, and R. Aguado, Phys. Rev. B 91, 045441 (2015).

48 A. Jellinggaard et al. (in preparation).

49 H. I. Jørgensen, T. Novotný, K. Grove-Rasmussen, K. Flensberg, and P. E. Lindelof, Nano Letters 7, 2441 (2007).

50 S. Csonka, L. Hofstetter, F. Freitag, S. Oberholzer, C. Schönenberger, T. S. Jespersen, M. Aagesen, and J. Nygård, Nano letters 8, 3932 (2008).

51 M. D. Schroer, K. D. Petersson, M. Jung, and J. R. Petta, Phys. Rev. Lett. 107, 176811 (2011). 